\title{
EL RENDIMIENTO ACADÉMICO DE LOS ESTUDIANTES VINCULADOS AL ROBO DE COMbustible. Región PUebla, MÉXICO ${ }^{1}$
}

\author{
THE ACADEMIC PERFORMANCE OF STUDENTS \\ linked to fuel theft. Puebla Region, Mexico
}

Rogelio Benítez Sánchez*

Adelina Espejel Rodríguez**

\section{* Maestro en Análisis Regional, Licenciado en Pedagogía. Universidad Pedagógica Nacional, Unidad 211, Puebla (México). rogelio.upn@gmail.com 0000-0001-9688-6990}

** Doctora en Ciencias Económicas por la Universidad de Camagüey, Cuba, Maestría en Análisis Regional por la UATx. Universidad Autónoma de Tlaxcala (UATx), ProfesorInvestigador. (México). adelinaer@hotmail.com 0000-0003-0299-796X

1 Investigación realizada entre agosto de 2017 y julio de 2020 en Puebla, México.

Cómo citar este artículo: Benítez, S. R. \& Espejel, R. A. (2021). El rendimiento académico de los estudiantes vinculados al robo de combustible. Región Puebla, México. Revista PACA 11, pp. 17-34.
Resumen: El objetivo de la investigación es conocer el rendimiento académico (RA) de los jóvenes de nivel secundario, vinculados con la extracción y venta ilegal de hidrocarburos, desde la perspectiva docente y del estudiante, antes y después de la llegada de los huachicoleros, en cinco municipios de la región centrooriente de Puebla. Se efectuó mediante una metodología cualitativa, donde se realizaron entrevistas y cuestionarios a docentes y a estudiantes de instituciones educativas de la región con altos índices de extracción ilegal de hidrocarburos directamente de los ductos de Petróleos Mexicanos (PEMEX). El alto índice delictivo en la región, aunado al aumento de jóvenes vinculados al crimen organizado, perturbó el ámbito educativo. Se encontró que el $70 \%$ de los docentes coincidieron en que hubo una disminución significativa en el RA y el $30 \%$ aseguró que no hubo cambios en su RA, pero todos coincidieron en que se presentó un cambio conductual. Se concluye que la extracción y venta ilegal de hidrocarburos ha trastocado el ámbito educativo y también el desempeño de los estudiantes, manifestándose bajo una intimidante posición de temor por parte del docente $y$, visto desde la perspectiva del estudiante, como un trabajo normalizado al cual debe darle prioridad, dando como resultado un rezago en la educación.

Palabras clave: huachicol, combustible, rendimiento, académico. 
Abstract: The objective of the research is to know the academic performance (AP) of high school youth linked to the illegal extraction and sale of hydrocarbons, from the teacher and student perspective, before and after the arrival of the huachicoleros, in five municipalities from the central eastern region of Puebla. It was carried out through a qualitative methodology where interviews and questionnaires were conducted with teachers and students from educational institutions, located in the region with high rates of illegal extraction of hydrocarbons directly from the pipelines of Petróleos Mexicanos (PEMEX). The high crime rate in the region, coupled with the increase in young people linked to organized crime, disturbed the educational environment, it was found that $70 \%$ of teachers agree that there was a significant decrease in AP and 30\% assured that there were no changes in their AP, but all agreed on a behavioral change. It is concluded that the illegal extraction and sale of hydrocarbons has disrupted the educational environment and also the performance of the students, manifesting itself under an intimidating position of fear on the part of the teacher and seen from the student's perspective as a standardized job to which priority should be given, resulting in a lag in education.

Keywords: huachicol, fuel, performance, academic.

\section{Introducción}

El robo de combustible a los ductos de Petróleos Mexicanos (PEMEX) por parte de organizaciones delictivas es un problema nacional; Puebla se encuentra entre los tres estados con mayor número de tomas aseguradas, y se tiene conocimiento de un aumento de la participación de estudiantes de nivel secundario, en estas operaciones de extracción clandestina. Estos adolescentes que cursan la secundaria están siendo contratados por el crimen organizado (EI Universal, 2017) y (UNIÓN Puebla, 2017a). Por tal motivo, el presente trabajo tuvo como objetivo conocer el rendimiento académico de los estudiantes de nivel secundario vinculados con la extracción y venta ilegal de hidrocarburos, desde la perspectiva docente y del propio estudiante, antes y después de la llegada de los huachicoleros [individuos y organizaciones dedicadas a la extracción y venta ilegal de hidrocarburos].

El trabajo se centra en ver el rendimiento académico más allá de las calificaciones numéricas, lo cual implica adentrarse en lo que pasa en el desempeño del estudiante día a día, y descubrir a través de la mirada del docente los aspectos importantes que marcan un cambio significativo antes y después de que el estudiante se vinculó al robo de hidrocarburos, 
sin dejar fuera la propia perspectiva que el estudiante tiene de sí mismo, sobre su desempeño y recuperar su testimonio como actor fundamental de este fenómeno social.

En los aspectos teóricos conceptuales se abordaron los términos con los que se define el rendimiento escolar, partiendo de las diferentes concepciones que existen al respecto. En los aspectos metodológicos se abordaron la población y la muestra, además de narrar de una forma puntual lo ocurrido con los instrumentos de investigación y la toma de decisiones que se realizaron para continuar con la investigación.

La sistematización y análisis de la información presentan un pequeño esbozo de cómo se organizó la información para finalmente darle paso a los resultados y la discusión, tomando en cuenta tanto la perspectiva del docente como la del estudiante y poder contrastar así sus diferentes posturas frente a un mismo fenómeno.

\section{Aspectos teóricos conceptuales}

Uno de los indicadores para verificar la efectividad del nivel adquirido en los aprendizajes es el rendimiento académico, el cual se define de diferentes maneras. Puede ser visto como constructo susceptible de adoptar valores cualitativos o cuantitativos que se aproximen a la evidencia de conocimientos desarrollados por el estudiante en el proceso de enseñanza aprendizaje (Edel, 2003).

El rendimiento académico al ser visto como una tabla de medición de los aprendizajes esperados "mide las capacidades del alumno, expresa lo que éste ha aprendido durante su proceso de aprendizaje y que al final los docentes expresan en una calificación aprobatoria o reprobatoria" (Hernández, 2014, p. 28).

Otros autores identifican el rendimiento académico como un resultado cuantitativo de 0 a 10 obtenido por el estudiante en el proceso de aprendizaje (Rivas, 2016), o puede convertirse en una expresión que permita conocer la existencia de calidad en la educación (Jara et al., 2008), o lograr hacer una medición de la eficiencia de los distintos insumos de la educación (Morales, Morales, y Holguín, 2016), también concebido como una "expresión valorativa del proceso educativo que [...] se expresa en 
el plano empírico mediante un conjunto de manifestaciones específicas, articuladas y relacionadas entre sí [...], su indicador es la suma total de calificaciones que se obtuvieron" (Fernández, Peña, y Vera, 2006).

Las diferentes concepciones respecto al rendimiento académico no sólo están en su definición, sino también en los factores que las ocasionan, Hernández (2014) identifica tres factores generales; familiar, personal y escolar (Tabla 1), que intervienen en el desempeño de los estudiantes.

Tabla 1

Factores y variables del rendimiento académico.

\begin{tabular}{ll}
\hline Factores & \multicolumn{1}{c}{ Variables } \\
\hline \multirow{3}{*}{ Familiar } & - El tipo de familia (nuclear, extensa u otra) \\
& - Situación económica en el hogar \\
& - El nivel educativo de los padres \\
& - Motivación (de la familia y de los profesores) \\
Personal & - La situación laboral del alumno \\
& - Tipo de materia (consideradas difíciles por los \\
& estudiantes) \\
& clases) \\
\hline
\end{tabular}

Fuente: Elaboración propia, basado en Hernández (2014).

El rendimiento académico como indicador de resultados tiene causalidades multifactoriales, lo que puede traducirse en influencias directa o indirectamente relacionadas entre sí, por lo cual se optó por apoyarse en lo propuesto por Reynoso (2011) que plantea, a partir del análisis de varios especialistas en el tema, la existencia y coincidencia de diferentes investigaciones, dividir tales causalidades en tres aspectos fundamentales: la efectividad de la institución educativa; el ambiente familiar; y la calidad de la comunidad.

La efectividad de la escuela tiene como función implícita que los estudiantes adquieran los aprendizajes esperados y tener un óptimo rendimiento académico mediante el uso de estrategias y herramientas 
que estimulen el nivel atencional de cualquier contenido educativo (ParraBolaños, Fidel, y de la Peña, 2017).

En el ambiente familiar, algunas investigaciones acusan que el nivel ocupacional y educativo que presentan los padres, son también variables determinantes en el momento de analizar el RA (Fajardo et al. 2017). Sin embargo, resulta imposible deslindar la influencia del apoyo familiar en el rendimiento académico del estudiante, resaltando nuevamente que existen matices que no se pueden explicar de forma general.

El contexto donde viven los estudiantes tiene gran influencia en su conducta, sus perspectivas y su educación. Esto se puede entender, como lo sugiere González (2003), derivado de la influencia de la zona en la que el estudiante vive, y de los efectos o consecuencias que resulten en la escuela por su localización, pues inciden inevitablemente en su rendimiento académico.

La finalidad de la exposición de los elementos ya mencionados es precisar que el RA es un "fenómeno multifactorial que puede ser explicado mediante la interacción de una serie de factores sobredeterminados" (Reynoso, 2011, p. 50), la calidad de la comunidad donde residen los estudiantes, se posicionará como una constante imprescindible de la presente investigación, siendo el crimen en el vecindario el punto de cohesión entre el estudiante y su RA.

Dada la existencia de diferentes definiciones sobre el rendimiento académico, se busca conocer la calificación cualitativa de los estudiantes, que el directivo y los profesores identificaron referentes al desempeño académico; por ello surge la necesidad de operacionalizar este concepto, y para entenderlo como el desempeño personal del estudiante en el proceso de enseñanza - aprendizaje desde la perspectiva del docente.

En cuanto al robo de combustible, de acuerdo con fuentes de PEMEX, tan solo en el último trimestre de 2015, el ducto conocido como Minatitlán - México, tenía una desviación de producto cercana a los 128 millones de litros de gasolina; la actividad ilegal se encontraba en el Estado de Puebla (Morales, 2017). 


\section{Aspectos metodológicos}

El método que se definió como marco para indagar sobre el objeto de estudio de la investigación fue el enfoque cualitativo. Se estudiaron los actores participantes dentro de su contexto como un todo para entender a la persona desde su marco de referencia.

\section{Población y muestra}

Las poblaciones determinadas fueron docentes y estudiantes de los planteles definidos en la región de estudio. Para el caso de los docentes se establecieron cuatro criterios de selección: a) Debían laborar en instituciones educativas de nivel secundario en cualquier modalidad, dentro de la región participante. b) Haber ejercido la docencia por más de tres años dentro del plantel donde prestan sus servicios profesionales. c) Conocer el significado de las palabras huachicoleros y/o halcón en los términos que denotan el crimen organizado. d) Tener conocimiento pleno de la existencia de los llamados huachicoleros.

Para definir la muestra de los estudiantes participantes en la investigación se consideró que debían ser aquellos identificados por los docentes con un vínculo en la actividad del huachicol y que no existiera riesgo para ninguno de ellos.

\section{Instrumentos}

Se utilizaron un cuestionario y una entrevista semiestructurada para obtener los datos en cuanto a la perspectiva del docente sobre el rendimiento académico de los estudiantes de nivel secundario. Ante el miedo a participar por parte de los docentes, se decidió aplicar los cuestionarios de forma electrónica, directamente a los e-mails de los docentes, y otro más estético para enviarse por mensajería instantánea como lo es la aplicación de WhatsApp. El cuestionario online fue enviado a alrededor de cien docentes, cuyo correo electrónico fue proporcionado por autoridades escolares; sin embargo, al cierre de la plataforma solo se tenía la respuesta de once docentes.

Para el análisis de la información se anexaron los siete cuestionarios obtenidos en la fase de piloteo, dado que la información presentada 
en ello era relevante. Además, se anexó un apartado de comentarios donde se vertieron datos importantes que no se tenían contemplados y representaron un hallazgo en la investigación.

Se diseñaron dos entrevistas, la primera abocada a docentes y la segunda a estudiantes. Pese a ser la perspectiva del docente el punto de interés, de los veinte contemplados para la entrevista, al final la mayoría se negó: solo cuatro docentes aceptaron ser entrevistados.

La segunda fase de la entrevista fue hecha a estudiantes que los docentes identificaron como colaboradores de los huachicoleros, los cuales, de manera voluntaria, aceptaron dar su postura en cuanto a su rendimiento escolar.

\section{Sistematización y análisis de la información}

Las entrevistas, cuestionarios y observaciones, nos permitieron detallar, sistematizar y analizar la información. Se usó el método de análisis descriptivo para expresar los resultados, acordes con los objetivos planteados en la presente investigación. Para ordenar la información se utilizó el programa Excel, que permitió organizar y categorizar los resultados. Se retomó a Strauss, y Corbin (2002, p. 20), que mencionan que "los datos cualitativos nos permiten relacionar conceptos con los datos brutos para luego organizar esquemas explicativos".

\section{Resultados y discusión}

\section{Aspectos generales de los participantes}

Los docentes que participaron en el cuestionario online fueron nueve hombres y nueve mujeres; uno fungía como directivo, una realizaba acciones de trabajo social, y los dieciséis restantes se desempeñaban como docentes frente a grupo. De los seis docentes que participaron en la entrevista, cinco son de género masculino y una del femenino; se tuvo en consideración una antigüedad igual o mayor a los tres años en la misma institución educativa; se distingue que dos fungen con cargo directivo; la media del servicio en la tarea educativa se ubica en los 20.6 años, lo que da cuenta de una amplia experiencia sobre la labor docente. 
En relación con los estudiantes, la media de edad es de catorce años, una es mujer y siete son hombres, cursan segundo y tercer año de secundaria y están identificados con un vínculo a los grupos huachicoleros. De los ocho participantes, hay un caso que no participa en la estructura de los huachicoleros; sin embargo, por vivir cerca de una toma de extracción de hidrocarburos, se ve afectado en diferente medida, por lo cual se consideró un testimonio valioso. Es indispensable precisar que los estudiantes valoran su relación con los procesos de extracción de combustible como un 'trabajo' y así será llamado, pese a ser un ilícito.

De la muestra de los estudiantes, tres son halcones; se les considera como espías que se ubican en lugares estratégicos para vigilar si algún camión militar, policías federales o carros desconocidos se acercan al lugar, con el fin de mantener controlados y despejados los caminos y rutas que usarán para el trasiego del combustible robado; el pago oscila entre los 500 y los 1000 pesos el día o turno (UNIÓN Puebla, 2017b). Dos estudiantes son copilotos, también llamados chalanes; son los que abren los contenedores y se encargan de taparlos cuando ya están llenos; también son los que están atentos a los radios y celulares para estar en contacto con los halcones y así agilizar la operación del conductor al momento del traslado; reciben por su trabajo una paga que oscila los 1200 y los 1500 pesos por cada viaje, de acuerdo con informantes clave; en un día pueden realizar hasta cuatro viajes. También se encontró que uno es ayudante de estaca, encargado de la recaudación de una renta fija por cada toma clandestina (Pérez, 2017). (Ver Tabla 2).

Tabla 2

Características generales de los estudiantes.

\begin{tabular}{llcl}
\hline Edad & Sexo & Grado escolar & Vínculo con el huachicol \\
\hline 13 & Masculino & $2^{\circ}$ & Halcón \\
13 & Masculino & $2^{\circ}$ & Familia vinculada \\
14 & Femenino & $2^{\circ}$ & Halcón \\
14 & Masculino & $2^{\circ}$ & Vivir frente a toma clandestina \\
14 & Masculino & $3^{\circ}$ & Halcón \\
15 & Masculino & $3^{\circ}$ & Copiloto \\
15 & Masculino & $3^{\circ}$ & Ayudante de estaca \\
15 & Masculino & $3^{\circ}$ & Copiloto \\
\hline
\end{tabular}

Fuente: Elaboración propia con base en el trabajo de campo. 
La percepción del RA desde la perspectiva de los docentes y los estudiantes

El rendimiento académico no puede ser relacionado con una sola perspectiva, pues existe un sinfín de factores que pueden afectar directa o indirectamente el desempeño del estudiante. Para entender un poco este universo multifactorial que incide en el RA, se les preguntó a los dieciocho docentes que participaron en el cuestionario cómo identifican el RA de los estudiantes (Tabla 3).

Tabla 3

Identificadores del rendimiento académico, usado por docentes.

Bajo RA detectado a partir de No. Alto RA detectado a partir de No.

\begin{tabular}{|c|c|c|c|}
\hline $\begin{array}{l}\text { Su desempeño y participación } \\
\text { en clase }\end{array}$ & 6 & $\begin{array}{l}\text { Su desempeño y participación } \\
\text { en clase }\end{array}$ & 6 \\
\hline $\begin{array}{l}\text { La atención diferenciada } \\
\text { (apático, tímido) }\end{array}$ & 4 & Su método de evaluación & 6 \\
\hline Sus métodos de evaluación & 3 & $\begin{array}{l}\text { Buen desempeño } \\
\text { competencial }\end{array}$ & 2 \\
\hline $\begin{array}{l}\text { No logra los aprendizajes } \\
\text { esperados }\end{array}$ & 2 & Resultados de PLANEA y SisAT & 1 \\
\hline $\begin{array}{l}\text { Los resultados de PLANEA y } \\
\text { SisAT }\end{array}$ & 1 & $\begin{array}{l}\text { Muestra de conocimientos } \\
\text { en plenaria }\end{array}$ & 1 \\
\hline Su historial académico & 1 & $\begin{array}{l}\text { Su forma de relacionarse } \\
\text { con los demás }\end{array}$ & 1 \\
\hline $\begin{array}{l}\text { Que no tiene metas y carece } \\
\text { de ideas }\end{array}$ & 1 & Un examen diagnóstico & 1 \\
\hline
\end{tabular}

Fuente: Elaboración propia con base en el trabajo de campo.

Los docentes identifican el RA mediante varios indicadores como son el desempeño y participación en clase, los métodos de evaluación, la atención diferenciada y los resultados SisAT. 
En cuanto al RA desde la mirada estudiantil, seis de los participantes indicaron su valoración como regular o bueno; usan expresiones como ni muy bien, ni muy mal o voy más o menos. Las razones que dan para estimar de esa forma su desempeño en el aula son variadas; por ejemplo, Rosita, de catorce años, (halcón) indica "Estoy en 7 y 8 [refiriéndose a las notas que obtienen] por lo que soy regular".

En tres casos se hace referencia a que desempeñan un trabajo y eso les reduce su capacidad para rendir de mejor forma en su desempeño escolar, tal como da cuenta el testimonio de Hugo, de quince años, (copiloto): "ahí voy más o menos, pues hay veces que... sí falto, pero casi nunca llego a faltar, aun cuando trabajo de noche; en clase tengo mucho sueño".

También hay quien asume tener un mal desempeño, derivado de las condiciones que se viven en su contexto, que le limitan para presentarse a la escuela:

Ya ni siquiera podemos salir [vive frente a una toma clandestina]; cuando vengo a la escuela, primero veo si no están [los huachicoleros], o le digo a mi mamá que si me puede traer. Hubo un día en que no vine porque se agarraron a balazos, me quedé vestido porque empezaron a darse los balazos allá abajo, pues mejor me quedé solo en casa, con mis carnales (Paco, catorce años, no vinculado).

Los estudiantes valoran que su rendimiento académico no ha cambiado después de entrar a "trabajar"; todos refieren que no han notado ninguna alteración, que no les afectó. Así lo sugiere Pablo, de quince años, (ayudante de estaca): "pues es lo mismo, no he sufrido ningún cambio". El RA también lo miden con los trabajos de clase, el cumplimiento de las tareas y no faltar ningún día.

Este rasgo compartido en los jóvenes puede deberse a que "el fracaso escolar no parecía ser un conflicto [...], muchos se percataban de que, a pesar de su mal desempeño, los profesores trataban de hacerlos pasar de año" (Kessler, 2007, p. 287).

Por otro lado, la perspectiva del estudiante que vive frente a la toma clandestina es diferente, al referir que ahora no se encuentra tranquilo y concentrado en la escuela. Al respecto, señala que la razón es porque 
está preocupado por su familia, ya que su padre ha increpado a los huachicoleros pidiendo los dejen en paz; añadió este recuerdo: "un día venía mi abuelita de compras, uno de ellos [huachicolero] venía en la camioneta, pues la pasaron a traer, pero ella rápidamente se fue a una orilla... pero se fue a un canal de agua" (Paco, catorce años).

En contraste con lo anterior está la mirada de los docentes, quienes poseen más elementos para entender y explicar el rendimiento académico de sus estudiantes, reflejado día a día en el aula. Alejandro (directivo), con 33 años de servicio, manifiesta que "si afecta... ya nada más están pensando en crecer, [...] juntarse con alguien más poderoso que ellos... para que les den una toma de huachicol, entonces ya lo del estudio lo están dejando a un lado".

Además, aseguró que existen tres factores que repercuten en su educación; el primero, las inasistencias continuas; en cuatro meses del ciclo escolar hay estudiantes con 26 faltas; esto debido a que reciben una llamada por su celular y deben acudir al momento; el segundo, siempre están pensando en cómo esquivar los peligros y no se concentran en clase; y el tercero, la ambición; hay estudiantes que ganan 13 mil pesos mensuales, más dinero del que su papá aporta a la familia; por consiguiente, algunos se convierten en el sostén de su casa.

Miriam, docente con 12 años de servicio, advierte que los adolescentes están perdiendo interés en la educación: "ven que sus papás se matan trabajando... y ganan 200 pesos..., ellos trabajan 2 horas y ganan 500 pesos, entonces [...], pueden adquirir este ingreso mayor... y no miden el peligro [...], no les interesa, ven la facilidad del dinero". Por lo tanto, se reconoce la existencia de estudiantes que, al llegar al colegio, pierden la motivación por continuar su formación escolar, desarrollando bajos niveles de bienestar y poca calidad en las interacciones personales. De acuerdo con Pinedo, Arroyo y Caballero (2017), estas actitudes suelen detonar en un descenso del rendimiento académico.

Otro docente, con 17 años de servicio, comenta respecto a los estudiantes cuyas familias están inmiscuidas en el huachicol y por esa situación hacen caso omiso del apoyo que necesita en casa para mejorar su nivel académico: 
Lo que podemos notar en los chavos es mucho abandono [...], vemos mucho descuido [de los padres] en cuanto a estar pendientes de las actividades académicas de sus hijos porque unos... inclusive se encuentran ya detenidos, y obviamente no hay ese nexo que se debe tener entre docentes y padres de familia (David, docente).

La existencia de algunos padres que se encuentren detenidos por la autoridad agrava la vida cotidiana de estos jóvenes, porque ahora su familia se ve fragmentada y dejan de tener un respaldo emocional que los acompañe en su desarrollo, esto a la situación de pobreza en la que viven.

Moreno (2001, p. 26) indica que "la realidad social está parcelada de manera casi impermeable, y el individuo se ve obligado a transitar por sus diferentes contextos habituales, adoptando en cada uno de ellos lo que podríamos denominar el disfraz adecuado", cumpliendo así diferentes roles, en la escuela, en casa, y en la comunidad y en su actividad delictiva.

Con una idea diferente se encuentra el directivo Jacobo, con 39 años de servicio, quien arguye que "no afecta, ya que él solamente identifica una familia que se dedicaba a eso, en el ciclo escolar pasado. De los tres hijos que tiene esa familia, dos se retiraron de la escuela y una si concluyó su formación de secundaria". Por otro lado, un docente de la misma institución, con 8 años de servicio, difiere de la inexistencia actual de estudiantes vinculados al huachicol en la secundaria. Comentó que, en ocasiones, estos llegan desvelados por su 'trabajo', pero cumplen con lo que se les pide:

Antes se les pedía tal material... y nos decían que no podían porque no contaban con el recurso suficiente... a lo mejor no tenían computadora en casa..., y ahorita la gran mayoría de esos alumnos ya tienen hasta internet en el celular, todo lo material lo adquieren más fácilmente (Manuel, docente).

El que un directivo y un docente de un mismo plantel den información diferente sobre el tema, da cuenta de que es un tema del que resulta difícil abordar por factores que desconocemos, pero podemos inferir uno, el miedo a las represalias.

Los docentes viven una experiencia degradada por los conflictos [...] y la necesidad de hacerse cargo de situaciones sociales para las que no se sienten preparados. Ellos y los directivos se preguntan qué hacer con los casos problemáticos; pocas son las reglas que quedan en pie; se intenta 
en general no expulsarlos ni excluirlos del sistema educativo, pero, al mismo tiempo, hay un nivel de conflictividad para el que carecen de recursos. El desdibujamiento de la ley también afecta de forma directa a las instituciones escolares (Kessler, 2004, p. 255).

En cuanto a las principales dificultades que observaron los docentes para el logro de los aprendizajes esperados y con ellos alcanzar un buen rendimiento académico de los estudiantes, se dividieron en dos posturas. Jacobo, (directivo, 39 años de servicio), aseguró: "acá cumplen con su obligación, que es el trabajo escolar, y ya si ellos se dedican a otra cosa, no afecta en nada a la institución". El estudiante es visto como un objeto donde la educación no tiene como fin la integración de la realidad que le rodea en los aprendizajes esperados; simplemente, es la incorporación de información sin la reflexión o la crítica respecto al mundo. La postura de este docente desde el inicio de la entrevista fue aceptar la presencia del huachicol en la región, pero negar cualquier efecto, tanto negativo como positivo, dentro de la institución.

En un posicionamiento opuesto se tiene a cinco docentes que coincidieron en que existía un cambio conductual en los estudiantes, el desinterés por asistir a clases y la preferencia a la huachicoleada [como nombran generalmente ese 'trabajo']; con ello, las implicaciones que representa trabajar de noche "el que no está dormido no presta atención, o reprueban, no traen tareas, en fin, no tienen ningún interés por su aprendizaje" (Miriam, docente, 12 años de servicio). Inclusive, de acuerdo con el directivo Alejandro (33 años de servicio) "vienen casi obligados por los padres, ya no tienen ganas ni interés por estudiar, porque ya tienen un ingreso extra... ya vienen sin ganas, nada más por cumplimiento, cumplen y mienten, ya no les interesa para nada la escuela".

Kessler indica una posibilidad de inclusión de estos jóvenes en una forma de acuerdo no formalizado entre ambas partes:

Mientras los alumnos no perjudicaran el orden en clase, los profesores no los 'molestaban', aunque estuvieran totalmente desconectados de las materias. [...] establecerse una suerte de pacto implícito de no interferencia entre alumnos y profesores que querían evitar todo tipo de conflictividad en la clase. [...] intentan llevarse bien con los profesores, para que la escuela sea lo más fácil posible, y tratan de tener una buena conducta para poder pasar sin estudiar nada o lo menos posible (Kessler, 2004, pp. 203-204). 
Para conocer mejor qué percepción tenían los docentes sobre los estudiantes que ya tienen identificados en el robo de combustible, se les preguntó cómo era el rendimiento académico de esos adolescentes antes de que ellos como docentes tuvieran conocimiento de la existencia de los huachicoleros, y cuál es la percepción de los mismos estudiantes hoy en día (Ver Tabla 4).

Tabla 4

Percepción docente del rendimiento académico de sus estudiantes.

\begin{tabular}{lrlr}
\hline $\begin{array}{l}\text { Antes de la llegada de los } \\
\text { huachicoleros }\end{array}$ & & $\begin{array}{l}\text { Identificados con vínculos al } \\
\text { huachicol }\end{array}$ \\
\hline Igual / sin cambios & 3 & Igual / sin cambios & 3 \\
Bajo & 1 & Bajo & 13 \\
Regular & 13 & Regular & 2 \\
Alto & 1 & Alto & 0 \\
\hline
\end{tabular}

Fuente: Elaboración propia con base en el trabajo de campo.

De los dieciocho docentes, una dijo que el estudiante tenía un rendimiento alto y después bajo, argumentando lo siguiente: "antes tenía otras aspiraciones o metas en la vida [...] hoy, por el hecho de tener dinero, se cree intocable" (Luisa, 11 años de servicio). Once docentes aseveraron, que antes eran regulares y hoy en día tienen un desempeño bajo. Entre las causas que dieron están: el dormir en clase, las inasistencias y el incumplimiento de tareas; pero hubo un docente que culpó a la familia por este hecho: "lamentablemente los padres aceptan dicha actividad y no existe el rigor o la orientación debida hacia sus hijos y los dejan hacer lo que quieran" (Alberto, docente, 8 años de servicio).

Finalmente hubo seis docentes que refieren que no existió cambio alguno, de estos estudiantes, uno tenía bajo rendimiento académico y continuaba igual; el docente Carlos ( 3 años de servicio) no dio explicación del porqué; tres dijeron que todo seguía igual bajo la argumentación de que: "el buen estudiante lo sigue siendo y el mal estudiante también" (María, docente, 20 años de servicio). Mientras los docentes Juan (18 años de servicio) y Sergio (3 años de servicio) aseguraban que seguían con un desempeño regular, gracias a que las mamás de estos estudiantes estaban vigilantes de su educación. 
Existen, además, valoraciones previas sobre los estudiantes que limitan su mejor desempeño, cayendo el docente en una profecía autocumplida debido a su percepción, confinándolos al efecto Pigmalión de convertir una afirmación subjetiva en realidad, como lo define Moreno (2010, p. 179); "las creencias sobre los alumnos buenos concluyen en resultados buenos y a la inversa. La creencia docente es generadora de actitudes, esfuerzo y desempeño".

El bajo rendimiento académico que perciben los docentes es atribuido al cambio conductual que sufrió el estudiante cuando incursionó en el mundo delictivo. Cabe destacar que esta conducta de violencia no es tan marcada al grado de significar un peligro para la comunidad escolar; también reconocieron la falta de participación de los padres de familia ante esta situación, pues existen familias completas dedicadas a esta actividad delictiva, dejando fuera de sus prioridades apoyar al docente y al estudiante a obtener los aprendizajes esperados para un mejor progreso de su desempeño escolar.

La familia también juega un papel fundamental en las decisiones de estos estudiantes, a tal grado de tomar como natural ciertos eventos delictivos por la tolerancia y complicidad que se vive en casa, al no increpar al adolescente por su actuación dentro y fuera del colegio, posiblemente rompiendo los mismos padres el límite disciplinar que con anterioridad pudieron regir en su hogar. Al respecto Gabriel Kessler sugiere que:

La actitud de la familia ante sus robos. [...] lejos de inquirir en demasía sobre el origen del dinero, su madre acepta calladamente [...]. Las relaciones pacíficas entre los habitantes del hogar, en suma, se basan en el silencio y en un pacto implícito de no intromisión en las actividades que el joven realiza más allá de los límites del hogar (Kessler, 2004, p. 157).

El estudiantado, por su parte, califica como rendimiento académico el acto de entregar una tarea o trabajo y asistir a clase; la mayoría reconoció que en ocasiones se quedaban dormidos en el aula, lo cual disminuye la proyección de rendimiento académico a una simple presencia corpórea constante en la escuela, lo suficiente para obtener una medición de estudiante regular.

Tienen la sensación de que la escuela se los quiere sacar de encima y prefiere hacerlos pasar de año, que terminen el ciclo, tanto para no perjudicarlos como para que abandonen rápidamente la institución [...]. La 
escuela, adopta una serie de arreglos de compromiso, ya que, al no poder controlarlos y al mismo tiempo intentan no expulsarlos del sistema sin el título, renuncian a toda exigencia con tal de que salgan lo antes posible de ahí. [...], la institución escolar 'no sirve absolutamente para nada', ya que, aun sin estudiar, logran no solo pasar de año sino incluso obtener el título (Kessler, 2004, p. 198).

A partir de los testimonios es posible apreciar la disminución del rendimiento académico de los estudiantes vinculados al huachicol de acuerdo con la perspectiva dada por los docentes participantes. Los cambios que detectaron con mayor frecuencia es la apatía, las conductas agresivas hacia sus pares $y$, en algunos casos, la falta de respeto a las autoridades educativas, ofreciendo sobornos o la venta de combustible robado. Pese a lo anterior, el estudiante se asume a sí mismo sin cambios en su desempeño académico.

\section{Conclusiones y hallazgos de la investigación}

Los estudiantes vinculados al huachicol, al ser parte de la economía del hogar y aportar sus ingresos para las necesidades básicas de sus integrantes, crea una nueva premisa que debe desmitificarse: el posible desvanecimiento del sistema de poder de la familia tradicional, la modificación de valores y reglas que rigen sus hogares, dejando algunas condicionantes como la presión constante para terminar sus estudios básicos como regla primordial; quizá por ello la existencia de sobornos hacia los docentes para no ser reprobados.

Los estudiantes vinculados a la estructura de los huachicoleros negaron haber bajado su rendimiento académico; para ellos todo sigue igual porque a pesar de sus constantes faltas o dormir en clase, argumentan que entregan los trabajos y tareas, aunque no así en tiempo y forma, lo cual denota una percepción errónea de su propio RA.

Finalmente, los hallazgos de esta investigación son un aporte válido para futuras investigaciones, ya que describe una problemática vigente donde los grupos criminales constantemente cambian de giro, pero mantienen la misma estructura de operación, apoyados en la corrupción y en la marginación, donde existe una alta vulnerabilidad en los adolescentes estudiantes que necesita ser visibilizada. 
De acuerdo con el Consejo Nacional de Evaluación de la Política de Desarrollo Social (CONEVAL), en el año 2019 existían en la región de estudio [franja de huachicol], 192.296 personas que vivían en zonas de atención prioritaria urbana o rural, lo cual denota que las zonas de marginación suelen ser los bastiones de reclutamiento más efectivos para la delincuencia organizada.

\section{Referencias Bibliográficas}

CONEVAL. (2019). Pobreza en México. Recuperado de https://www.coneval. org.mx/Medicion/Paginas/PobrezaInicio.aspx

Edel, R. (2003). El rendimiento académico: concepto, investigación y desarrollo. REICE. Revista Electrónica Iberoamericana sobre Calidad, Eficacia y Cambio en Educación, 1(2). Recuperado de http://www.redalyc.org/articulo. oa? $\mathrm{id}=55110208$

El universal. (2017). Entérate. ¿Qué es un huachicolero?. Recuperado de https:// www.eluniversal.com.mx/articulo/estados/2017/05/4/enterate-que-es-unhuachicolero

Fajardo, F., Maestre, M., Felipe, E., León, B., y Polo, M. I. (2017). Análisis del rendimiento académico de los alumnos de secundaria obligatoria según las variables familiares. Educación XXI, 20(1), 209-232. https://doi. org/10.5944/educXX1.14475

Fernández, J, Peña, A., y Vera, F. (2006). Los estudios de trayectoria escolar, su aplicación en la educación media superior. Graffylia, 6, 24-29.

González, C. (2003). Factores determinantes del bajo rendimiento académico en educación secundaria. Universidad Complutense de Madrid.

Hernández, A. (2014). Trayectorias escolares en estudiantes del CBTiS No. 212. Una mirada a los factores del rendimiento escolar. Universidad Autónoma de Tlaxcala, México.

Jara, D., Velarde, H., Gordillo, G., Guerra, G., León, I., Arroyo, C., y Figueroa, M. (2008). Factores influyentes en el rendimiento académico de estudiantes del primer año de medicina. Anales de la Facultad de Medicina, 69(3)

Kessler, G. (2004). Sociología del delito amateur. Buenos Aires, Argentina: Paidós.

Kessler, G. (2007). Escuela y delito juvenil. La experiencia educativa de jóvenes en conflicto con la ley. Revista Mexicana de Investigación Educativa, 12(032), 283-303. Recuperado de http://www.comie.org.mx/documentos/ rmie/v12/n032/pdf/N032N.pdf

Morales, L., Morales, V., y Holguín, S. (2016). Rendimiento Escolar. Revista Electrónica de humanidades, tecnología y ciencia del IPN, 1-5.

Morales, P. (2017, enero 4). Por qué Puebla es el paraíso para los huachicoleros. HuffPost México. Recuperado de https://www. 
huffingtonpost.com.mx/2017/01/04/por-que-puebla-es-el-paraiso-para-loshuachicoleros_a_21646909/

Moreno, F. X. (2001). Análisis psicopedagógico de los alumnos de educación secundaria obligatoria con problemas de comportamiento en el contexto escolar. Universitat Autònoma de Barcelona.

Moreno, P. (2010). La política educativa de la globalización. México. IPN. Recuperado en: http://xplora.ajusco.upn.mx:8080/jspui/ handle/123456789/515

Parra-Bolaños, N., Fidel, M., y de la Peña, C. (2017). Atención y Memoria en estudiantes con bajo rendimiento académico. Un estudio exploratorio. Reidocrea, 6(7), 74-83. Recuperado de http://www.ugr.es/ reidocrea/6-7. pdf

Pérez, F. (2017, marzo 2). Hasta $\$ 12$ mil pesos pagan a niño como ‘halcón' de huachicoleros. Excelsior. Recuperado de http://www.excelsior.com.mx/ nacional $/ 2017 / 03 / 02 / 1149672$

Pinedo, R., Arroyo, M. J., y Caballero, C. (2017). Afectividad positiva y negativa en el futuro docente: relaciones con su rendimiento académico, salud mental y satisfacción con la vida. Contextos educativos: Revista de Educación, 20(20), 11-26.

Reynoso, E. L. (2011). Factores que determinan el rendimiento escolar en el nivel secundario en el Estado de Nuevo León. Universidad Autónoma de Nuevo León. Recuperado de http://eprints.uanl.mx/2730/6/1080089642.pdf\%5Cn

Rivas, V. (2016). El trabajo en equipo y la relación con el rendimiento escolar en educación básica. Universidad Pedagógica Nacional, Unidad 211, Puebla, México.

SEP (2017). Modelo educativo para la educación obligatoria. Ciudad de México: Secretaría de Educación Pública.

Strasuss, A., y Corbin, J. (2002). Bases de la investigación cualitativa. Técnicas y procedimientos para desarrollar la teoría fundamentada. Colombia: Universidad de Antioquia.

UNIÓN Puebla. (2017a, marzo 13). Maestros ya no quieren trabajar por miedo a huachicoleros. UNIÓN Puebla.

UNIÓN Puebla. (2017b, mayo 25). Halcones de huachicoleros ganan mil pesos por noche. UNIÓN Puebla. Recuperado de http://www.unionpuebla.mx/ articulo/2017/05/25/seguridad/halcones-de-huachicoleros-ganan-milpesos-por-noche 